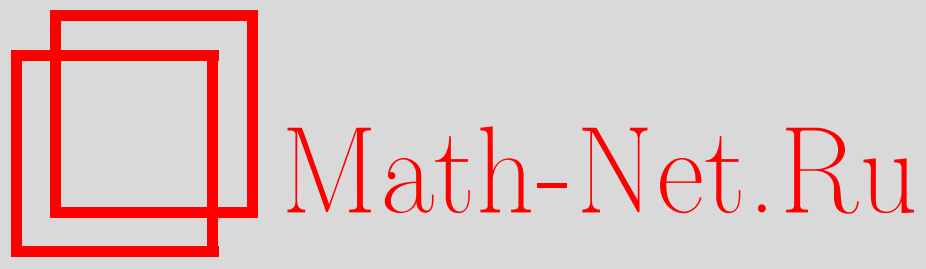

В. П. Маслов, В. Е. Назайкинский, Туннельный канонический оператор в термодинамике, Функи. анализ и его прил., 2006, том 40, выпуск 3, 12-29

DOI: https://doi.org/10.4213/faa740

Использование Общероссийского математического портала Math-Net.Ru подразумевает, что вы прочитали и согласны с пользовательским соглашением

http://www .mathnet.ru/rus/agreement

Параметры загрузки:

IP: 54.198 .55 .26

26 апреля 2023 г., 16:53:44

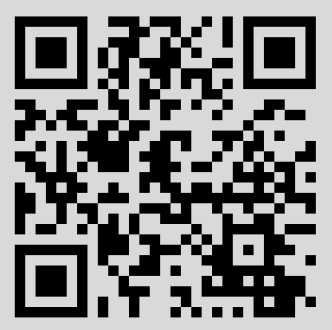


Функииональный анализ и его приложения

2006, т. 40, вып. 3, с. 12-29

УДК $517.9+536.7$

\title{
Туннельный канонический оператор в термодинамике
}

\author{
(c) 2006. В. П. МАслов, В. Е. НАЗАЙКИНСКиЙ
}

\section{$\S 1$. Введение}

Давно известно о существовании параллелей между термодинамикой и классической механикой (см., например, [1]). Формулы метода термодинамических потенциалов (см., например, [10]), выражающие термодинамические переменные через производные термодинамического потенциала по двойственным «естественным» переменным, с точностью до обозначений тождественны формулам, выражающим в классической механике импульсы как производные производящей функции по координатам [5], а переход от одних наборов естественных переменных к другим (или, что то же самое, от одного термодинамического потенциала к другому) есть не что иное, как преобразование Лежандра. Термодинамические переменные разбиваются на пары сопряженных переменных (давление-объем, энтропия-температура, число частиц-химический потенциал), и множество равновесных состояний термодинамической системы представляет собой в пространстве термодинамических переменных лагранжсево многообразие (это понятие было введено одним из авторов [3]), задаваемое термодинамическими потенциалами как производящими функциями.

Лагранжевы многообразия естественно возникают и в совсем другой задаче о квазиклассической асимптотике решений уравнения Шрёдингера. Квазиклассические решения доставляются в этой задаче квантованием лагранжева многообразия, лежащего на энергетическом уровне классического гамильтониана, т. е. сопоставлением ему класса быстроосциллирующих при $h \rightarrow 0$ функций, среди которых и находятся отвечающие данному уровню энергии квазиклассические решения. Квантование это задается каноническим оператором [3].

В статистической физике, как и в квантовой механике, имеется естественный малый параметр. Это $1 / N$, где $N$ - число частиц. В сочетании с тем фактом, что в термодинамике, которая представляет собой «классический предел» статистической физики при $N \rightarrow \infty$, имеются естественные лагранжевы многообразия, это наводит на мысль, что и в этой ситуации квантование лагранжева многообразия должно приводить к приближению по параметру, стремящемуся к нулю, на этот раз в статистической физике. Эта идея подтверждается известной формулой [1]

$$
F(v, T)=\lim _{N \rightarrow \infty}(k T / N) \ln \Sigma
$$

для удельной свободной энергии, где $k$ - постоянная Больцмана, $v-$ удельный объем, $T$ - температура, а $\Sigma$ - статистическая сумма. Из этой формулы получается, что при больших $N$

$$
\Sigma \sim e^{-N F(v, T) / k T} .
$$


Формула (1) стоит к общему квантованию лагранжева многообразия в термодинамике в таком же отношении, как ВКБ-приближение - к каноническому оператору.

Идея квантования термодинамического лагранжева многообразия была развита в работах [6], [7]. Отметим, что это квантование весьма сильно отличается от своего квантовомеханического аналога. Там речь идет о быстроосциллирующих функциях, в изучаемой же нами ситуации никаких осцилляций нет, а рассматриваются быстроубывающие функции, в простейшем случае - вида правой части соотношения (1). Поэтому вместо обычного канонического оператора рассматривается так называемый туннельный канонический оператор [4]. В квантовой механике, как известно, все переменные равноправны; волновая функция в координатном представлении унитарно эквивалентна волновой функции в импульсном представлении (эквивалентность задается преобразованием Фурье). В термодинамике каждая пара сопряженных переменных содержит экстенсивную переменную (т. е. такую, значение которой в данном состоянии пропорционально количеству вещества; примером могут служить объем, внутренняя энергия, энтропия и т. д.) и интенсивную переменную (значение которой не зависит от количества вещества, как, например, у температуры и давления). В [6] было предложено в качестве координат рассматривать интенсивные переменные, а в качестве импульсов - экстенсивные, причем интенсивные переменные играют выделенную роль: для квантования термодинамического лагранжева многообразия следует использовать туннельный оператор, значения которого являются функции именно от координат. Логарифм функции, задаваемой туннельным каноническим оператором на термодинамическом лагранжевом многообразии, был назван статистическим потенциалом, и основной постулат заключается в том, что туннельный канонический оператор задает асимптотику статистической суммы при больших $N$, так что статистический потенциал точнее описывает свойства термодинамической системы, чем термодинамические потенциалы.

Разумеется, в термодинамике наряду с феноменологической квазиклассикой, или геометрическим квантованием в духе Н. Бора, имеется и задача о квазиклассическом предельном переходе, т.е. о строгом вычислении асимптотики статистической суммы при $N \rightarrow \infty$. В модельных примерах иногда удается, записав выражение для статистической суммы в виде бесконечномерного интеграла по траекториям (см. Фейнман-Хибс [2], а также [8]), вычислить асимптотику этого интеграла в неособом случае при $N \rightarrow \infty$ методом Лапласа, что позволяет получить не только экспоненту, но и предэкспоненциальный множитель, а из последнего - и меру на лагранжевом многообразии. В общем же случае эта задача является значительно более сложной и далека от решения. В данной статье мы не будем на этом останавливаться.

Заметим, что представление с помощью туннельного канонического оператора сразу же приводит к правилу Максвелла равных площадей для фазовых переходов I рода. А именно, если термодинамическое лагранжево многообразие $L$ в пространстве переменных $(p, v)$ (давление-объем) при проекции на координатную ось $p$ имеет несколько ветвей над данной точкой $p_{0}$, локально диффеоморфно проектирующихся на окрестность точки $p_{0}$, то статистический потенциал будет задаваться формулой

$$
G(p, N)=k T \ln \left\{\sum_{j} a_{j}(p) e^{-\frac{N}{k T} \mu_{j}(p)}\right\},
$$


где сумма берется по всем ветвям $j$ многообразия $L$ над точкой $p_{0}$. При $N \rightarrow \infty$ «выживает» лишь экспонента с наименьшим значением потенциала Гиббса $\mathscr{G}=$ $N \mu(p)$. «Переход» с одной ветви на другую происходит при совпадении значений, а правило равных площадей получается из соотношения $d \mathscr{G}=V d p$.

При этом в отличие от классической термодинамики, где переход с одной ветви на другую происходит скачкообразно, статистический потенциал дает более плавный переход, с большими, но конечными производными. Далее, в окрестности тех точек, где касательная к лагранжеву многообразию вертикальна («тройная точка» $)^{1)}$, классическая феноменологическая термодинамика по существу неприменима (например, как отмечают Ландау и Лифшиц [1], вычисления на ее основе приводят формально к бесконечным флуктуациям, а при машинном счете алгоритмы становятся в этой точке неустойчивыми к вычислительным погрешностям). Замена в окрестности таких точек термодинамического потенциала статистическим потенциалом, получаемым из туннельного канонического оператора, устраняет расходимости, вызванные неприменимостью классических формул в этой точке, и позволяет вычислить истинные флуктуации ${ }^{2)}$. Фактически формулы туннельного канонического оператора в окрестности особенности проектирования сводятся к введению «контрфлуктуации»: лагранжево многообразие сдвигается на малое время $\sigma$ вдоль фазового потока гамильтониана ${ }^{3)}$ $\boldsymbol{p}^{2} / 2$ (что устраняет особенность проектирования), а затем туннельное ВКБвыражение на вновь полученном многообразии свертывается с фундаментальным решением уравнения теплопроводности за то же время $\sigma$.

В данной статье рассматривается следующий круг вопросов.

1. Пусть асимптотика статистической суммы задается туннельным каноническим оператором. Основным необходимым в приложениях объектом является не сама эта функция, а ее логарифм (статистический потенциал) и его производные. Поэтому мы изучаем, с какой точностью канонический оператор дает асимптотику этих производных.

2. Выражение для туннельного канонического оператора, отвечающего термодинамическому лагранжеву многообразию, в работе [6] имеет в неособой карте вид $\exp \{-N G / k T\} \varphi$, т. е. фазой экспоненты является не само действие $G$ (потенциал Гиббса) на лагранжевом многообразии, а частное $G / T$ от его деления на одну из координат, температуру ${ }^{4)}$. Хотя соответствующие формулы и дают правильный ответ, это наводит на мысль, что координаты на лагранжевом многообразии выбраны не вполне удачно. Чтобы перейти к «правильным» координатам, мы трактуем наше лагранжево многообразие как лежандрово, делаем в контактном фазовом пространстве контактную замену переменных и снова возвращаемся к лагранжевой трактовке. В результате энтропия (которая ранее была одной из фазовых переменных) становится термодинамическим потенциалом, а действием на новом лагранжевом многообразии оказывается преобразование Лежандра от энтропии, известное в термодинамике как функиия

1) В данной статье мы не касаемся метастабильных состояний.

2) Вопросу о флуктуациях будет посвящена другая работа.

3)Здесь $(\boldsymbol{q}, \boldsymbol{p})$ - импульсы и координаты в фазовом пространстве; мы обозначаем их полужирными буквами, чтобы избежать путаницы с обозначением $p$ для давления.

4) Постоянная $k$, разумеется, несущественна для наших рассуждений, ибо большим параметром можно считать $N / k$. 
Планка, которая задается формулой $H=G / T$. Переход от таких фазовых переменных к обычным можно трактовать как введение переменных действие-угол. Заметим, что новые «координаты» и «импульсы» не вполне обычны; так, в качестве координат (интенсивных переменных) выступают теперь функции $\beta=1 / T$ (обратная температура) и $\beta p$, где $p$ - давление. В неравновесной термодинамике и статистической физике всегда говорят о возрастании энтропии или о принципе наивыгоднейшего энергетического состояния. Мы покажем, что это эквивалентные вещи. Эквивалентность распространяется и на минимум термодинамического потенциала Гиббса, но без касательного преобразования это на первый взгляд не согласуется с общим принципом возрастания энтропии.

3. В конструкцию туннельного канонического оператора входит мера на лагранжевом многообразии. В [6] вопрос о выборе меры не обсуждался (тем более, что в первом приближении в неособых картах она не влияет на статистический потенциал). Тем не менее вопрос о существовании на термодинамическом лагранжевом многообразии естественной меры представляет значительный интерес. В физике имеется естественный объект - так называемые кинетические коэффичиенты, - который, как мы увидим, приводит к естественно определенной римановой метрике на лагранжевом многообразии. Если термодинамическую систему вывести из равновесия с окружающей средой, то она будет возвращаться по своему лагранжеву многообразию к состоянию равновесия вдоль траектории наискорейшего (относительно длины, задаваемой этой метрикой) возрастания совокупной энтропии. По метрике естественным образом определяется и мера. Эта мера, как и метрика, определена лишь с точностью до мультипликативной константы, которая не оказывает влияния на производные логарифма статистической суммы. Если кинетические коэффициенты в окрестности особых точек растут, то соответствующий вклад в статистический потенциал может быть существенным.

4. В заключение мы кратко обсуждаем вопрос о конструкции термодинамического лагранжева многообразия, отвечающего смеси веществ. При этом могут возникать лагранжевы многообразия с особенностями. Построение туннельного канонического оператора на таких многообразиях выходит за рамки данной работы.

\section{§2. Туннельный канонический оператор}

2.1. Лагранжево многообразие, действие и мера. Введем объекты, участвующие в определении туннельного канонического оператора. Пусть $\Lambda$ лагранжево многообразие в фазовом пространстве $\mathbb{R}_{\boldsymbol{q}, \boldsymbol{p}}^{2 n}$, снабженном симплектической формой $\omega^{2}=\sum_{i=1}^{n} d \boldsymbol{p}_{i} \wedge d \boldsymbol{q}_{i}$. Предполагается, что выполнено условие

$$
\oint_{\gamma} \boldsymbol{p} d \boldsymbol{q}=0 \text { для произвольного замкнутого контура } \gamma \subset \Lambda
$$

(это всегда так для многообразий, отвечающих термодинамике), так что форма $\boldsymbol{p} d \boldsymbol{q}$ на $\Lambda$ имеет однозначную первообразную $S$ (функцию действия), определенную (если $\Lambda$ связно) с точностью до аддитивной константы, которую мы как-либо зафиксируем. Пусть $\pi: \Lambda \rightarrow \mathbb{R}_{\boldsymbol{q}}^{n}$ - проекция многообразия $\Lambda$ на координатное пространство $\mathbb{R}_{\boldsymbol{q}}^{n}$. Будем предполагать, что проекция собственнал, т. е. прообраз любого компактного множества компактен. 
ОПРЕДЕЛЕНИЕ 1. Точку $\alpha \in \Lambda$ назовем равновесной, если

$$
S(\alpha)=\min _{\beta \in \pi^{-1}(\alpha)} S(\beta),
$$

и неравновесной в противном случае.

Далее, пусть на $\Lambda$ задана гладкая вещественная мера $\mu$, т. е. нечетная форма объема. В локальной системе координат $\left(\alpha_{1}, \ldots, \alpha_{n}\right)$ на $\Lambda$ она имеет вид $\mu=$ $\mu_{\alpha}\left|d \alpha_{1} \cdots d \alpha_{n}\right|$, а при заменах координат плотность меры умножается на модуль якобиана:

$$
\mu_{\alpha}=\mu_{\beta}\left|\operatorname{det} \frac{\partial\left(\beta_{1}, \ldots, \beta_{n}\right)}{\partial\left(\alpha_{1}, \ldots, \alpha_{n}\right)}\right|
$$

\section{2. Локальное определение туннельного оператора.}

2.2.1. Туннельный оператор вблизи неособой точки. Точка $\alpha_{0} \in \Lambda$ называется неособой, если проекция $\pi$ является диффеоморфизмом в ее достаточно малой окрестности $U$.

ОПреДЕЛЕНИЕ 2. Туннельный канонический оператор в окрестности $U$ неособой точки $a_{0}$ задается формулой

$$
\left[K_{0} \varphi\right](x)=\left.\left\{e^{-\lambda S(\alpha)} \varphi(\alpha) \sqrt{\mu_{\boldsymbol{q}}(\alpha)}\right\}\right|_{\pi(\alpha)=x}, \quad \varphi \in C_{0}^{\infty}(U) .
$$

Здесь $\lambda \rightarrow+\infty-$ большой параметр (в термодинамике в качестве этого параметра выступает $\lambda=N / k)$, а $\mu_{\boldsymbol{q}}$ - плотность меры $\mu$ в координатах $\left(\boldsymbol{q}_{1}, \ldots, \boldsymbol{q}_{n}\right)$.

2.2.2. Туннельный оператор вблизи особой точки. Пусть $\alpha_{0} \in \Lambda$ - особая точка (дифференциал $d \pi$ вырождается в $\alpha_{0}$ ). Сделаем каноническую замену переменных так, чтобы точка $\alpha_{0}$ стала неособой, определим туннельный оператор в новых координатах формулой (3) и вернемся к старым координатам, применив к полученному выражению «проквантованное» обратное каноническое преобразование. Дадим теперь строгое определение. Для этого рассмотрим гамильтониан

$$
H_{\sigma}=\frac{1}{2} \sum_{j=1}^{n} \sigma_{j} \boldsymbol{p}_{j}^{2}, \quad \text { где } \sigma=\left(\sigma_{1}, \ldots, \sigma_{n}\right), \sigma_{j} \geqslant 0-\text { константы, }
$$

и зададим каноническое преобразование $g_{\sigma}$ как сдвиг на время -1 вдоль траекторий соответствующей системы Гамильтона

$$
\dot{\boldsymbol{q}}_{j}=\sigma_{j} \boldsymbol{p}_{j}, \quad \dot{\boldsymbol{p}}_{j}=0, \quad j=1, \ldots, n,
$$

так что $g_{\sigma}(\boldsymbol{q}, \boldsymbol{p})=(\boldsymbol{q}-\sigma \boldsymbol{p}, \boldsymbol{p})$.

ПРЕДЛОЖЕНИЕ 3. Множество точек $\sigma$, для которых $\alpha_{0}$ - неособая точка лагранжева многообразия $g_{\sigma}(\Lambda)$, непусто, открыто и содержит сколь угодно малье по норме векторы б из положительного октанта.

ДокАЗАтельство. Рассмотрим максимальный линейно независимый набор дифференциалов переменных $\boldsymbol{q}_{j}$ в точке $\alpha_{0}$; без ограничения общности можно считать, что это $d \boldsymbol{q}_{1}, \ldots, d \boldsymbol{q}_{k}$. Тогда в силу лагранжевости независимы дифференциалы $d \boldsymbol{q}_{1}, \ldots, d \boldsymbol{q}_{k}, d \boldsymbol{p}_{k+1}, \ldots, d \boldsymbol{p}_{n}$, а значит (так как набор $d \boldsymbol{q}_{1}, \ldots, d \boldsymbol{q}_{k}$ максимален), и дифференциалы $d \boldsymbol{q}_{1}, \ldots, d \boldsymbol{q}_{k}, d\left(\boldsymbol{q}_{k+1}-\sigma_{k+1} \boldsymbol{p}_{k+1}\right), \ldots, d\left(\boldsymbol{q}_{n}-\sigma_{n} \boldsymbol{p}_{n}\right)$ при любых $\sigma_{k+1}, \ldots, \sigma_{n}>0$. 
Действие на многообразии $\Lambda_{\sigma}=g_{\sigma}(\Lambda)$ ( $\sigma$-действие) зададим формулой

$$
S_{\sigma}(\alpha)=S(\alpha)-\frac{1}{2} \sum_{j=1}^{n} \sigma_{j} \boldsymbol{p}_{j}^{2}
$$

а меру - формулой $\mu_{\sigma}=\left(g_{\sigma}^{-1}\right)^{*}(\mu)$.

Зафиксируем такое $\sigma$, что точка $\alpha_{0}$ - неособая точка многообразия $\Lambda_{\sigma}$; тогда «неособый» туннельный оператор $\widetilde{K}_{\sigma}$ на $\Lambda_{\sigma}$ корректно определен в малой окрестности $U$ точки $\alpha_{0}$.

ОПРЕДЕЛЕНИЕ 4. Туннельный оператор на $\Lambda$ в окрестности $U$ особой точки $a_{0}$ задается формулой

$$
K_{\sigma} \varphi=e^{\lambda \widehat{H}_{\sigma}}\left(\widetilde{K}_{\sigma} \varphi\right), \quad \varphi \in C_{0}^{\infty}(U)
$$

где проквантованный гамильтониан $\widehat{H}_{\sigma}$ задается формулой

$$
\widehat{H}_{\sigma}=\frac{1}{2} \sum_{j=1}^{n} \sigma_{j}\left(-\lambda^{-1} \frac{\partial}{\partial x_{j}}\right)^{2}
$$

Заметим, что экспонента $e^{\lambda \widehat{H}_{\sigma}}$ в $(7)$ корректно определена (как решение уравнения теплопроводности) и представляет собой интегральный оператор с ядром

$$
G_{\sigma}(x, y)=\prod_{j=1}^{n} \sigma_{j}^{-1}\left(\frac{\lambda}{2 \pi}\right)^{n} \exp \left\{-\lambda \sum_{j=1}^{n} \frac{\left(x_{j}-y_{j}\right)^{2}}{2 \sigma_{j}}\right\} .
$$

(Здесь и далее для краткости мы проводим все рассуждения и выписываем формулы только для случая, когда все $\sigma_{j}$ положительны. Общий случай отличается лишь более громоздкими обозначениями.)

2.2.3. $\sigma$-преобразование. Отметим, что если обе функции действия $S$ и $S_{\sigma}$ в некоторой области лагранжева многообразия можно выразить через соответствующие неособые координаты $x=\boldsymbol{q}$ и $\widetilde{x}=\boldsymbol{q}-\sigma \boldsymbol{p}$, то они связаны аналогом преобразования Лежандра. Пусть $S(x), x \in \mathbb{R}^{n},-$ гладкая вещественная функция с ограниченными производными, а $\sigma$ - вектор с компонентами $\sigma_{j}>0$, $j=1, \ldots, n$.

ОПРЕДЕЛЕНИЕ 5. $\sigma$-преобразованием функции $S(x)$ назовем функцию

$$
S_{\sigma}(y)=\max _{x}\left\{S(x)-\frac{(x-y)^{2}}{2 \sigma}\right\}, \quad \text { где } \quad \frac{(x-y)^{2}}{2 \sigma} \stackrel{\text { def }}{=} \sum_{j=1}^{n} \frac{\left(x_{j}-y_{j}\right)^{2}}{2 \sigma_{j}} .
$$

ТеОрема 6. При достаточно малъх $\sigma_{1}, \ldots \sigma_{n}>0$-преобразование функиии $S(x)$ представляет собой гладкую функиию от $y$, а функиия $S(x)$ можсет быть восстановлена по $S_{\sigma}(y)$ с помощъю обратного б-преобразования

$$
S(x)=\min _{y}\left\{S_{\sigma}(y)+\frac{(x-y)^{2}}{2 \sigma}\right\} \text {. }
$$

ДокАзАТЕЛьСтво. Можно представить $\sigma$-преобразование как композицию

$$
S(x) \longmapsto F(x)=S(x)-\frac{x^{2}}{2 \sigma} \stackrel{\text { преобразование }}{\stackrel{\text { Лежандра }}{\longmapsto}} \widetilde{F}(y) \longmapsto S_{\sigma}(y)=\widetilde{F}(y)-\frac{y^{2}}{2 \sigma}
$$


трех преобразований, где преобразование Лежандра задается формулой

$$
\widetilde{F}(y)=\max _{x}\{F(x)+x y / \sigma\} .
$$

При малых $\sigma_{1}, \ldots \sigma_{n}>0$ функция $F(x)=S(x)-x^{2} / 2 \sigma$ имеет отрицательно определенный гессиан $\partial^{2} F(x) / \partial x^{2}$, т. е. строго вогнута. Тогда ее преобразование Лежандра выпукло и бесконечно гладко, и обратное преобразование Лежандра

$$
F(x)=\min _{y}\{\widetilde{F}(y)-x y / \sigma\}
$$

приводит к исходной функции $F(x)$. Теорема доказана с учетом того факта, что обратное $\sigma$-преобразование также может быть разложено в композицию

$$
S_{\sigma}(y) \mapsto \widetilde{F}(y)=S_{\sigma}(y)+\frac{y^{2}}{2 \sigma} \underset{\text { зование Лежандра }}{\stackrel{\text { обратное преобра- }}{\longmapsto}} F(x) \mapsto S(x)=F(x)+\frac{x^{2}}{2 \sigma} \text {. }
$$

2.3. Глобальное определение туннельного оператора. Теперь из введенных выше «локальных» определений мы составим глобальное. Пусть $Г \subset \Lambda$ - множество равновесных точек (оно замкнуто в силу собственности проекции $\pi$ ). Рассмотрим локально конечное покрытие множества Г открытыми множествами $U_{j} \subset \Lambda$, каждому из которых сопоставлен набор $\sigma(j) \in \mathbb{R}_{+}^{n}$, такой, что туннельный оператор $K_{\sigma(j)}$ определен в окрестности $U_{j}$. Далее, пусть $\left\{e_{j}\right\}$ - разбиение единицы на $\Gamma$, подчиненное покрытию $U_{j}$, a $\left\{\psi_{j}\right\}$ - локально конечная система срезающих функций на $\mathbb{R}^{n}$, такая, что $\psi_{j}(x) \equiv 1$ в некоторой окрестности множества $\pi\left(U_{j} \cap \Gamma\right)$.

ОПРЕДЕЛЕНИЕ 7. Туннельный канонический оператор $K$ на пространстве $C^{\infty}\left(\Lambda \times[0,1]_{h}\right)$ функций $\varphi(\alpha, h)$, гладко зависящих от точки $\alpha \in \Lambda$ и параметра $h \in[0,1]$, задается формулой

$$
[K \varphi](x, \lambda)=\sum_{j} \psi_{j}(x) K_{\sigma(j)}\left(e_{j}(\alpha) \varphi\left(\alpha, \lambda^{-1}\right)\right)
$$

где локальные операторы $K_{\sigma}$ имеют вид $(3)$ при $\sigma=0$ (т. е. в неособом случае) и (7) при $\sigma \neq 0$ (т. е. в окрестностях особых точек).

Заметим, что сумма (16) локально конечна. Обозначим через $R$ множество функций, представимых при достаточно больших $\lambda$ в виде (16) для какого-либо $\varphi \in C^{\infty}\left(\Lambda \times[0,1]_{h}\right)$ при каком-либо выборе покрытий, наборов $\sigma(j)$, разбиения единицы и срезающих функций.

Теорема 8 [4]. Если $K$ и $K^{\circ}$ - два туннелъных оператора, определенных с помощъю разных покрытий, наборов $\sigma(j)$, разбиений единищы и систем срезающих функций, удовлетворяющих описанным выше условиям, то

$$
K \varphi-K^{\circ} \varphi \in \lambda^{-1} R \quad \text { для любого } \varphi \in C^{\infty}\left(\Lambda \times[0,1]_{h}\right) .
$$

ДокАЗАтЕльство. Достаточно проверить, что если локальные туннельные операторы $K_{\sigma}$ и $K_{\widetilde{\sigma}}$ определены в области $U$ и $\widetilde{\sigma} \leqslant \sigma$ (покомпонентно), то

$$
K_{\sigma} \varphi=K_{\widetilde{\sigma}}\left(\varphi+\lambda^{-1} \varphi_{1}\right)
$$


для любой функции $\varphi \in C_{0}^{\infty}(U)$, где $\varphi_{1}$ гладко зависит от малого параметра $\lambda^{-1}$ вплоть до нуля. При $\widetilde{\sigma}=0$ это следует из метода Лапласа (нужные соотношения для стационарной точки выводятся из того факта, что действия $S$ и $S_{\sigma}$ получаются друг из друга $\sigma$-преобразованием). Случай же $\widetilde{\sigma} \neq 0$ легко сводится к предыдущему с помощью цепочки равенств

$$
K_{\sigma} \varphi=e^{\lambda \widehat{H}_{\sigma}}\left(\widetilde{K}_{\sigma} \varphi\right)=e^{\lambda \widehat{H}_{\widetilde{\sigma}}} e^{\lambda \widehat{H}_{\sigma-\widetilde{\sigma}}}\left(\widetilde{K}_{\sigma} \varphi\right)=e^{\lambda \widehat{H}_{\widetilde{\sigma}}}\left(\widetilde{K}_{\widetilde{\sigma}}\left(\varphi+\lambda^{-1} \varphi_{1}\right)\right),
$$

где второе равенство справедливо в силу группового свойства разрешающего оператора уравнения теплопроводности, а третье доказывается с помощью преобразования, связывающего многообразия $\Lambda_{\sigma}$ и $\Lambda_{\widetilde{\sigma}}$, и уже рассмотренного случая $\tilde{\sigma}=0$.

ЗАмЕчАниЕ 9. Из теоремы 8 вытекает, что в областях пространства $\mathbb{R}_{x}^{n}$, для которых в $\pi^{-1}(x)$ имеется единственная равновесная точка, причем лежащая в неособой карте, туннельный оператор определен на не обращающихся в нуль функциях $\varphi$ с «мультипликативной» точностью: при переходе к другому выбору произвольных элементов, входящих в определение туннельного оператора, функция $K \varphi$ умножается на $1+O\left(\lambda^{-1}\right)$. В общем случае это не так.

2.4. Производные статистического потенциала. Основной интерес в термодинамике представляет не сам туннельный оператор, а производные $P=$ $\partial \Phi / \partial x$ задаваемого им «статистического потенциала»

$$
\Phi(x, \lambda)=-\lambda^{-1} \ln K \varphi,
$$

где функция $\varphi$ на лагранжевом многообразии всюду положительна ${ }^{1)}$. Изучим вопрос о точности, с которой эти производные не зависят от произвола в конструкции туннельного оператора. Рассмотрим сначала простейший случай: данной точке $x_{0} \in \mathbb{R}^{n}$ соответствует единственная равновесная точка $\alpha \in \pi^{-1}\left(x_{0}\right)$, лежащая в неособой карте на $\Lambda$. Тогда вблизи $x_{0}$ имеем $K \varphi=e^{-\lambda S(x)} a(x)$, где $a(x)=\sqrt{\mu_{q}(x)} \varphi(x)$, а $S(x)$ - действие на многообразии $\Lambda$, выраженное в координатах $x$, и

$$
P(x, \lambda)=\frac{\partial S(x)}{\partial x}-\lambda^{-1} a^{-1}(x) \frac{\partial a(x)}{\partial x} .
$$

Функция $P(x, \lambda)$ ограничена вместе со всеми производными равномерно по $\lambda$. Если вместо неособого туннельного оператора использовать особый с некоторым $\sigma$, получая вместо $P$ новую функцию $\widetilde{P}$, то, как следует из результатов предыдущего раздела, его можно свести к неособому оператору с амплитудой $a+\lambda^{-1} b$ вместо $a$, где $b-$ некоторая гладкая функция, и нетрудно подсчитать, что $\widetilde{P}-P=O\left(\lambda^{-2}\right)$ (равномерно вместе с производными). Однако уже для случая двух равновесных точек $\alpha_{1}, \alpha_{2} \in \pi^{-1}\left(x_{0}\right)$ (что в термодинамике соответствует фазовому переходу I рода) ситуация в окрестности точки $x_{0}$ будет другой. А именно (штрихами обозначены производные по $x$ ),

$$
P(x, \lambda)=\frac{e^{-\lambda S_{1}(x)}\left[S_{1}^{\prime}(x) a_{1}(x)-\lambda^{-1} a_{1}^{\prime}(x)\right]+e^{-\lambda S_{2}(x)}\left[S_{2}^{\prime}(x) a_{2}(x)-\lambda^{-1} a_{2}^{\prime}(x)\right]}{e^{-\lambda S_{1}(x)} a_{1}(x)+e^{-\lambda S_{2}(x)} a_{2}(x)},
$$

1) На самом деле в термодинамике $\varphi \equiv 1$, но нам удобно рассмотреть чуть более общий случай. 
где индексы 1 и 2 отвечают двум существенным ветвям лагранжева многообразия над $x_{0}$. Функция $P(x, \lambda)$ по-прежнему непрерывна и равномерно по $\lambda$ ограничена, но в малой окрестности «поверхности Стокса» $S_{1}(x)=S_{2}(x)$ она резко переходит со значения $P(x, \lambda) \approx \partial S_{1}(x) / \partial x$ на $P(x, \lambda) \approx \partial S_{2}(x) / \partial x$, так что в этой окрестности ее первые производные могут быть порядка $\lambda$. (Более того,

$$
\left|\partial^{\beta} P / \partial x^{\beta}\right| \leqslant C_{\beta} \lambda^{|\beta|}, \quad|\beta|=0,1,2, \ldots,
$$

причем показатель степени в оценке в общем случае улучшить нельзя.) Переход к особому каноническому оператору опять сводится к замене амплитуд $a_{j}$ на $a_{j}+\lambda^{-1} b_{j}, j=1,2$, и вычисления показывают, что

$$
\left|\partial^{\beta}(\widetilde{P}-P) / \partial x^{\beta}\right| \leqslant C_{\beta} \lambda^{|\beta|-1} .
$$

Таким образом, в этом случае туннельный оператор определяет производные статистического потенциала с «относительной точностью» $O\left(\lambda^{-1}\right)$.

Изучим теперь общий случай, когда над точкой $x_{0}$ может находиться произвольное (конечное) число существенных ветвей лагранжева многообразия, причем предположение о неособости этих ветвей не делается.

Теорема 10. Пусть $\varphi-$ гладкая положительная функиия на $\Lambda$. Тогда производные $P=\partial \Phi / \partial x$ статистического потенииала (17) удовлетворяют оценкам (18) и корректно определенъ с точностью до функиий, удовлетворяющих оценкам (19) на любом компакте в $\mathbb{R}_{x}^{n}$.

ДокАЗАтЕльство. Поскольку утверждение теоремы локально, достаточно рассмотреть малую окрестность некоторой точки $x_{0} \in \mathbb{R}^{n}$. В этой окрестности

$$
K \varphi=\sum_{j=1}^{m} K_{\sigma(j)} \varphi \equiv \sum_{j=1}^{m} I_{j}\left[a_{j}\right]
$$

где суммирование ведется по всем существенным ветвям лагранжева многообразия над $x_{0}, a_{j}=\varphi \sqrt{\mu_{j}}$ на соответствующей ветви лагранжева многообразия, a $I_{j}[a]:=K_{\sigma(j)}\left[a / \sqrt{\mu_{j}}\right]$. Справедлива формула коммутации

$$
-\lambda \frac{\partial}{\partial x} I_{j}[a]=I_{j}\left[\frac{\partial S_{j}}{\partial y} a-\lambda^{-1} \frac{\partial a}{\partial y}\right],
$$

где $S_{j}$ есть $\sigma$-действие, а $y$ - неособая координата после $\sigma$-преобразования. Поэтому (обозначая производную штрихом) имеем

$$
P(x, \lambda)=\frac{I_{1}\left[S_{1}^{\prime} a_{1}-\lambda^{-1} a_{1}^{\prime}\right]+\cdots+I_{m}\left[S_{m}^{\prime} a_{m}-\lambda^{-1} a_{m}^{\prime}\right]}{I_{1}\left[a_{1}\right]+\cdots+I_{m}\left[a_{m}\right]} .
$$

Для положительной функции $а$ справедлива очевидная оценка

$$
\left|I_{j}[f a]\right| \leqslant I_{j}[a] \sup _{\operatorname{supp} a}|f| .
$$

Используя ее, получаем ограниченность функции (21), т. е. оценку (18) при $\beta=0$. Дифференцируя функцию (21) с учетом формулы (20) и снова пользуясь неравенством (22), получаем оценку (18) в общем случае. Чтобы доказать оценку $(19)$, заметим, что переход от $P$ к $\widetilde{P}$, как и ранее, сводится к замене амплитуд $a_{j}$ на $a_{j}+\lambda^{-1} b_{j}$. Остается вычислить разность соответствующих функций $(21)$ и оценить ее и ее производные, пользуясь соотношениями (20) и (22). 
ЗАмЕчАНиЕ 11. Главный член асимптотики для разности $\widetilde{P}-P$ имеет вид

$$
\begin{gathered}
\lambda^{-1}\left\{\frac{\left(I_{1}\left[S_{1}^{\prime} b_{1}\right]+\cdots+I_{m}\left[S_{m}^{\prime} b_{m}\right]\right)\left(I_{1}\left[a_{1}\right]+\cdots+I_{m}\left[a_{m}\right]\right)}{\left(I_{1}\left[a_{1}\right]+\cdots+I_{m}\left[a_{m}\right]\right)^{2}}\right. \\
\left.-\frac{\left(I_{1}\left[S_{1}^{\prime} a_{1}\right]+\cdots+I_{m}\left[S_{m}^{\prime} a_{m}\right]\right)\left(I_{1}\left[b_{1}\right]+\cdots+I_{m}\left[b_{m}\right]\right)}{\left(I_{1}\left[a_{1}\right]+\cdots+I_{m}\left[a_{m}\right]\right)^{2}}\right\},
\end{gathered}
$$

что в частных случаях приводит к оценкам, более сильным, чем (19). Например, если $m=1$ и существенная точка неособая, то главный член равен нулю и мы получаем $\widetilde{P}-P=O\left(\lambda^{-2}\right)$, как и было указано выше для этого случая.

\section{§3. Классическая термодинамика}

\section{1. Аналогия между термодинамикой и классической механикой.} Напомним прежде всего нужные сведения из классической механики.

В ее гамильтоновой формулировке набор переменных $z$, определяющих состояние системы (фазовую точку), подразделяется на координатъ $\left(\boldsymbol{q}_{1}, \ldots, \boldsymbol{q}_{n}\right)$ и сопряженные к ним импульсъь $\left(\boldsymbol{p}_{1}, \ldots, \boldsymbol{p}_{n}\right), z=\left(\boldsymbol{q}_{1}, \ldots, \boldsymbol{q}_{n}, \boldsymbol{p}_{1}, \ldots, \boldsymbol{p}_{n}\right)$. Действие определяется затем как интеграл от формы $\boldsymbol{p} d \boldsymbol{q}=\sum_{j=1}^{n} \boldsymbol{p}_{j} d \boldsymbol{q}_{j}$,

$$
S=S_{0}+\int_{\gamma} \boldsymbol{p} d \boldsymbol{q},
$$

где $S_{0}$ - постоянная интегрирования. Вообще говоря, этот интеграл зависит от выбора пути интегрирования $\gamma$. Если же в фазовом пространстве системы выделить поверхность $L$, на которой форма $\boldsymbol{p} d \boldsymbol{q}$ замкнута, то интеграл, по крайней мере локально, будет зависеть только от начальной и конечной точек $\alpha_{0}$ и $\alpha_{1}$ пути $\gamma$. Размерность поверхности $L$ не превосходит $n$. В случае максимальной размерности $\operatorname{dim} L=n$ (только этот случай нас и интересует) поверхность называется лагранжевым многообразием. На лагранжевом многообразии формула (23) определяет (в общем случае многозначную) функцию действия

$$
S(\alpha)=S_{0}+\int_{\alpha_{0}}^{\alpha} \boldsymbol{p} d \boldsymbol{q},
$$

где интеграл берется по произвольному контуру $\gamma$, соединяющему начальную точку $\alpha_{0}$ и точку $\alpha$ на поверхности $L$. (Константу $S_{0}$ в $(24)$ обычно выбирают равной нулю, поскольку произвол в константе интегрирования уже обеспечивается выбором начальной точки.)

В простейшем случае, когда поверхность $L$ диффеоморфно проектируется на координатную $\boldsymbol{q}$-плоскость и, следовательно, задается уравнениями вида $\boldsymbol{p}=$ $\boldsymbol{p}(\boldsymbol{q})$, действие, определенное формулой (24), можно выразить через координаты $\boldsymbol{q}$, и тогда многообразие $L$ задается уравнениями

$$
\boldsymbol{p}_{j}=\frac{\partial S}{\partial \boldsymbol{q}_{j}}\left(\boldsymbol{q}_{1}, \ldots, \boldsymbol{q}_{n}\right), \quad j=1, \ldots, n .
$$

В общем случае $L$ не проектируется диффеоморфно на $\boldsymbol{q}$-плоскость, но в окрестности любой точки $\alpha \in L$ всегда можно в качестве локальных координат выбрать набор функций вида

$$
\left(\boldsymbol{q}_{I}, \boldsymbol{p}_{\bar{I}}\right) \equiv\left\{\boldsymbol{q}_{j}, \boldsymbol{p}_{k}: j \in I, k \in \bar{I}=\{1, \ldots, n\} \backslash I\right\}
$$


для некоторого подмножества индексов $I \subset\{1, \ldots, n\}$. В этом случае многообразие $L$ в окрестности точки $\alpha$ задается уравнениями вида

$$
\boldsymbol{p}_{I}=\frac{\partial S_{I}}{\partial \boldsymbol{q}_{I}}\left(\boldsymbol{q}_{I}, \boldsymbol{p}_{\bar{I}}\right), \quad \boldsymbol{q}_{\bar{I}}=-\frac{\partial S_{I}}{\partial \boldsymbol{p}_{\bar{I}}}\left(\boldsymbol{q}_{I}, \boldsymbol{p}_{\bar{I}}\right),
$$

где $S_{I}\left(\boldsymbol{q}_{I}, \boldsymbol{p}_{\bar{I}}\right)$ - функция $S_{I}(\alpha)=S(\alpha)-\sum_{j \in \bar{I}} \boldsymbol{p}_{j}(\alpha) \boldsymbol{q}_{j}(\alpha)$, выраженная в координатах $\left(\boldsymbol{q}_{I}, \boldsymbol{p}_{\bar{I}}\right)$. Несложно проверить, что для тех точек лагранжева многообразия, где как набор $\left(\boldsymbol{q}_{1}, \ldots, \boldsymbol{q}_{n}\right)$, так и набор $(26)$ можно взять в качестве локальных координат, функции $S(\boldsymbol{q})$ и $S_{I}\left(\boldsymbol{q}_{I}, \boldsymbol{p}_{\bar{I}}\right)$ связаны преобразованием Лежсандра по переменным с индексами $j \in \bar{I}$.

Вернемся к термодинамике. Переменные состояния термодинамической системы подразделяются на интенсивные (которые при объединении нескольких систем в одну стремятся в процессе установления термодинамического равновесия принять некоторое общее значение) и экстенсивные (которые при объединении систем просто складываются), причем интенсивные и экстенсивные переменные образуют пары; некоторые из них показаны в следующей таблице:

\begin{tabular}{|c|c|}
\hline интенсивные переменные & сопряженные экстенсивные переменные \\
\hline температура $T$ & минус энтропия $-S$ \\
давление $p$ & объем $V$ \\
химический потенциал $\mu$ & минус число частиц $-N$ \\
угловая скорость $\Omega$ & момент импульса $M$ \\
$\ldots$ & $\ldots$ \\
\hline
\end{tabular}

ЗАмЕчАниЕ. (а) Энтропия и число частиц со знаком минус отвлекают от механико-термодинамической аналогии, делая ее менее прозрачной.

(b) На самом деле, как мы увидим ниже, рассмотренные выше переменные скорее сродни переменным действие-угол классической механики.

Система в состоянии равновесия характеризуется термодинамическими потенииалами, которые представляют собой экстенсивные величины, являющиеся функциями некоторого «естественного» набора (для каждого потенциала - своего) «независимых» термодинамических переменных. Остальные («зависимые») термодинамические переменные определяются как производные потенциалов по независимым переменным. Например, внутренняя энергия $U$ зависит от естественных переменных $S, V, N$ и ее дифференциал задается формулой

$$
d U(S, V, N)=T d S-p d V+\mu d N
$$

Аналогично, для энтальпии $H=U+p V$, свободной энергии $F=U-T S$ и термодинамического потенциала Гиббса $G=F+p V$ имеем

$$
\begin{aligned}
& d H(S, p, N)=T d S+V d p+\mu d N \\
& d F(T, V, N)=-S d T-p d V+\mu d N \\
& d G(T, p, N)=-S d T+V d p+\mu d N
\end{aligned}
$$


так что, например, в терминах термодинамического потенциала Гиббса уравнения состояния термодинамической системы имеют вид ${ }^{1)}$

$$
S=-\frac{\partial G}{\partial T}, \quad V=\frac{\partial G}{\partial p}, \quad \mu=\frac{\partial G}{\partial N} .
$$

Отметим, что ни в одном из потенциалов в число аргументов не входят одновременно какая-либо интенсивная переменная и сопряженная к ней экстенсивная переменная.

Из приведенных выше формул видно, что многообразие возможных состояний термодинамической системы есть просто лагранжево многообразие в фазовом пространстве, в котором роль физических координат играют интенсивные переменные, а роль импульсов - сопряженные к ним экстенсивные переменные.

Более точно, определим псевдокоординатъь ${ }^{2)} \boldsymbol{q}$ и сопряженные к ним псевдоимпульсы $\boldsymbol{p}$, полагая

$$
\boldsymbol{q}=(T, p, \mu), \quad \boldsymbol{p}=(-S, V,-N) .
$$

Тогда множество $L$ возможных состояний термодинамической системы представляет собой трехмерное лагранжево многообразие в шестимерном фазовом пространстве $\mathbb{R}_{\boldsymbol{q}, \boldsymbol{p}}^{6}$, а термодинамические потенциалы суть просто функции $S_{I}$ для различного выбора подмножества $I$, задающие многообразие $L$ согласно формуле (27). При этом различные термодинамические потенциалы связаны между собой преобразованиями Лежандра по соответствующим переменным.

ЗАмЕчАниЕ 12. Поскольку при «удвоении» системы экстенсивные величины увеличиваются в два раза, а интенсивные переменные не меняются вовсе, лагранжево многообразие $L$ однородно по экстенсивным переменным: если $(\boldsymbol{q}, \boldsymbol{p}) \in L$, то и $(\boldsymbol{q}, \lambda \boldsymbol{p}) \in L$ при любом $\lambda>0$. Поэтому лагранжево многообразие $L$ никогда не проектируется однозначно на псевдокоординатную плоскость (плоскость интенсивных переменных), и соответственно каждый из термодинамических потенциалов содержит в качестве аргумента хотя бы одну экстенсивную переменную (в приведенных выше примерах это $N$ ). Более того, действие на $L$ тождественно равно нулю (или произвольной постоянной), поскольку в силу однородности (тождество Эйлера) равна нулю на многообразии $L$ форма $\boldsymbol{p} d \boldsymbol{q}$. Таким образом, термодинамические потенциалы, которые получаются из действия вычитанием произведений вида $\boldsymbol{p}_{i} \boldsymbol{q}_{i}$, заведомо являются глобально однозначно определенными функциями на всем многообразии $L$.

В практических приложениях нередко фиксируют какую-либо из независимых переменных, т. е. переходят к лагранжеву многообразию $\widetilde{L}$ в пространстве меньшего числа измерений. Вновь полученное многообразие уже не будет однородным (если зафиксирована одна из экстенсивных переменных) и может (локально или глобально) диффеоморфно проектироваться на плоскость интенсивных переменных. Например, если зафиксировать число частиц, то лагранжево многообразие, задаваемое термодинамическим потенциалом Гиббса, диффеоморфно проектируется на двумерную плоскость псевдокоординат $(T, p)$.

1) В работах по термодинамике в таких случаях принято писать, например, $S=$ $[-\partial G / \partial T]_{V, N}$, имея в виду, что производная по $T$ берется при постоянных $V$ и $N$; это обозначение, впрочем, излишне, поскольку постоянство остальных аргументов при вычислении производной по данному аргументу входит в определение частных производных.

2) Мы пользуемся приставкой «псевдо», чтобы не возникало путаницы с обычными координатами и импульсами классической механики. 
3.2. Лежандрово представление термодинамики. Наряду с потенциалами типа перечисленных выше, в термодинамике используются также термодинамические потенциалы иного рода, переход к которым уже не может быть описан в терминах преобразования Лежандра. Типичным примером такого потенциала является сама энтропия $S$ :

$$
d S(U, V, N)=\frac{d U}{T}+\frac{p}{T} d V-\frac{\mu}{T} d N \equiv \beta d U+\beta p d V-\beta \mu d N,
$$

где $\beta=1 / T$ - обратная температура. Чтобы включить в наше описание и такого рода потенциалы тоже, перейдем от лагранжева описания к лежандрову, а именно, вместо $2 n$-мерного фазового пространства $\mathbb{R}_{\boldsymbol{q}, \boldsymbol{p}}^{2 n}$ рассмотрим $(2 n+1)$ мерное фазовое пространство $\mathbb{R}_{\boldsymbol{q}, \boldsymbol{p}, u}^{2 n+1}$ и вложим в него наше многообразие $L$ согласно уравнениям

$$
\boldsymbol{q}=\boldsymbol{q}(\alpha), \quad \boldsymbol{p}=\boldsymbol{p}(\alpha), \quad u \equiv 0
$$

(координата $u$ соответствует действию на нашем лагранжевом многообразии, которое в силу приведенного выше замечания тождественно равно нулю). Пространство $\mathbb{R}_{\boldsymbol{q}, \boldsymbol{p}, u}^{2 n+1}$ снабжено контактной структурой (т. е. классом эквивалентности 1-формы $\omega=d u-\boldsymbol{p} d \boldsymbol{q}$ относительно умножения на гладкие положительные функции), и при вложении (35) многообразие $L$ является лежандровым (т.е. форма $\omega$ равна нулю на $L)$.

Выпишем некоторые сопряженные интенсивные и экстенсивные переменные в этом представлении.

\begin{tabular}{|l|l|}
\hline интенсивные переменные & $\begin{array}{l}\text { сопряженные экстенсивные } \\
\text { переменные }\end{array}$ \\
\hline обратная температура $\beta=1 / T$ & $\begin{array}{l}\text { внутренняя энергия } U \\
\text { объем } V \\
\text { давление, деленное на температуру } \beta p \\
\text { химпотенциал, деленный на температуру } \beta \mu \\
\text { угловая скорость, деленная на температуру } \beta \Omega \\
\ldots\end{array}$ \\
$\begin{array}{l}\text { мину число частиц }-N \\
\text { момент импульса } M\end{array}$ \\
\hline
\end{tabular}

3.3. Контактные многообразия. Напомним общие определения.

ОПРЕДЕЛЕНИЕ 13. Однородным контактным многообразием называется многообразие $X$ размерности $2 n+1$, снабженное гладким действием группы $\mathbb{R}_{+}$положительных чисел и классом эквивалентности (относительно умножения на гладкие положительные функции) 1-формы $\omega$, которая является однородной степени 1 относительно действия группы $\mathbb{R}_{+}$и удовлетворяет условию максимальной неинтегрируемости $\operatorname{rank} \omega \wedge d \omega=2 n+1$.

Гладкое отображение $f: X_{1} \rightarrow X_{2}$ однородных контактных многообразий называется однородным контактным, если оно коммутирует с действием группы $\mathbb{R}_{+}$и $f^{*}\left(\omega_{2}\right)=a \omega_{1}$, где $a-$ положительная функция.

Канонической системой координат на однородном контактном многообразии $X$ называется такая система координат $(\boldsymbol{q}, \boldsymbol{p}, u)$, что все координатные функции однородны степени 0 или 1, u однородна степени 1 и форма $d u-\boldsymbol{p} d \boldsymbol{q}$ лежит в выделенном классе контактных форм на $X$.

Лежандровым многообразием в $X$ называется $n$-мерное гладкое подмногообразие $L \subset X$, такое, что контактные формы равны нулю на $L$. 
Будем называть однородные функции степеней 0 и 1 на $X$ экстенсивными и интенсивными величинами соответственно.

ПРЕДЛОЖЕНИЕ 14. Если $L$ - лежандрово подмногообразие в $X$, то в некоторой окрестности каждой точки $\alpha \in L$ существует такая каноническая система координат $(\boldsymbol{q}, \boldsymbol{p}, u)$, что в этой окрестности многообразие $L$ диффеоморфно проектируется на $\boldsymbol{q}$-плоскость.

В этих терминах можно дать следующую формулировку феноменологической термодинамики.

Множество равновесных состояний термодинамической системъ есть однородное леэсандрово подмногообразие $L$ в $\mathbb{R}^{2 n+1}$. Представления многообразия L с помощью термодинамических потенциалов соответствуют каноническим системам координат, выбранным в соответствии с предложсением 14. При этом координаты $\boldsymbol{q}$ являются независимыми переменными термодинамического потенииала, а сам термодинамический потенииал представляет собой функцию $u$, выражсенную через координаты $\boldsymbol{q}, u=u(\boldsymbol{q})$. Переход от одних термодинамических потенииалов к другим задается однородными контактными преобразованиями пространства $\mathbb{R}^{2 n+1}$.

3.4. Энтропия как действие. Фазовый переход I рода. Переход от задания термодинамики лагранжевым многообразием, на котором роль действия играет, скажем, внутренняя энергия (28), к такому заданию, в котором роль действия играет сама энтропия ${ }^{1)}$, может быть естественным образом произведен в рамках лежандрова формализма, развитого в предыдущем разделе. А именно, если задана внутренняя энергия $U$ как функция от переменных $S, V, N$, то термодинамическое лагранжево многообразие $L$ в пространстве переменных $\mathbb{R}_{\boldsymbol{q}, \boldsymbol{p}}^{6}$,

$$
\boldsymbol{q}=(T, p, \mu), \quad \boldsymbol{p}=(-S, V,-N),
$$

описывается уравнением

$$
d U=T d S-p d V+\mu d N, \quad \text { или } \quad \boldsymbol{q}=-\partial U / \partial \boldsymbol{p} .
$$

Вложим $L$ в контактное пространство $\mathbb{R}_{\boldsymbol{q}, \boldsymbol{p}, u}^{7}$, снабженное контактной формой $\alpha_{(1)}=d u+\boldsymbol{q} d \boldsymbol{p}$, с помощью отображения $L \ni(\boldsymbol{p}, \boldsymbol{q}) \longmapsto(\boldsymbol{q}, \boldsymbol{p}, U(\boldsymbol{p}))$ (т. е. «недостающая» седьмая компонента задается действием - в данном случае внутренней энергией - на $L$ ). Образ $L_{c}$ этого вложения представляет собой контактное многообразие относительно формы $\alpha_{(1)}$ в силу (36). Разделив теперь обе части уравнения (36) на $T$, получим $d S=T^{-1} d U+p T^{-1} d V-\mu T^{-1} d N$, или, вводя обозначение $\beta=1 / T$ («обратная температура»),

$$
d S=\beta d U+(\beta p) d V-(\beta \mu) d N .
$$

Если положить

$$
\widetilde{\boldsymbol{q}}=(\beta, \beta p, \beta \mu), \quad \widetilde{\boldsymbol{p}}=(U, V,-N), \quad \widetilde{u}=-S,
$$

то переход от переменных $(\boldsymbol{q}, \boldsymbol{p}, u)$ к переменным $(\widetilde{\boldsymbol{q}}, \widetilde{\boldsymbol{p}}, \widetilde{u})$ задается формулами

$$
\widetilde{\boldsymbol{q}}_{1}=\boldsymbol{q}_{1}^{-1}, \quad \widetilde{\boldsymbol{q}}_{2}=\boldsymbol{q}_{1}^{-1} \boldsymbol{q}_{2}, \quad \widetilde{\boldsymbol{q}}_{3}=\boldsymbol{q}_{1}^{-1} \boldsymbol{q}_{3}, \quad \widetilde{\boldsymbol{p}}_{1}=u, \quad \widetilde{\boldsymbol{p}}_{2}=\boldsymbol{p}_{2}, \quad \widetilde{\boldsymbol{p}}_{3}=\boldsymbol{p}_{3}, \quad \widetilde{u}=\boldsymbol{p}_{1},
$$

1) Заметим, что такое задание представляется наиболее естественным в силу фундаментальной роли, которую играет энтропия в описании термодинамических состояний и процессов. 
и непосредственная выкладка показывает, что

$$
d \widetilde{u}+\widetilde{\boldsymbol{q}} d \widetilde{\boldsymbol{p}}=d p_{1}+\boldsymbol{q}_{1}^{-1} d u+\boldsymbol{q}_{1}^{-1} \boldsymbol{q}_{2} d \boldsymbol{p}_{2}+\boldsymbol{q}_{1}^{-1} \boldsymbol{q}_{3} d \boldsymbol{p}_{3}=\boldsymbol{q}_{1}^{-1}(d u+\boldsymbol{q} d \boldsymbol{p}) .
$$

Иначе говоря, эта замена переменных является контактным преобразованием и, следовательно, переводит лежандрово многообразие $L_{c} \subset \mathbb{R}_{\boldsymbol{q}, \boldsymbol{p}, u}^{7}$ в лежандрово многообразие $\widetilde{L}_{c} \subset \mathbb{R}_{\widetilde{\boldsymbol{q}}, \tilde{\boldsymbol{p}}, \widetilde{u}}^{7}$ в пространстве переменных «с волной». Проектируя это многообразие в симплектическое пространство $\mathbb{R}_{\boldsymbol{q}, \boldsymbol{p}}^{6}$, получаем новое термодинамическое лагранжево многообразие в координатах $(\beta, \beta p, \beta \mu)$ (псевдокоординаты) и $(U, V,-N)$ (псевдоимпульсы), действие на котором с точностью до знака равно энтропии (см. (37)).

ЗАмЕчАниЕ 15. Отметим, что описанный выше переход от одного типа термодинамических координат к другим может рассматриваться как аналог перехода к координатам «действие-угол» в классической механике в том смысле, что функция, игравшая роль действия в одной из систем координат, становится одной из независимых переменных в новой системе координат. Разумеется, аналогия на этом пока что заканчивается, ибо в классической механике угловые переменные выделяются условием цикличности (т. е. независимости гамильтониана от этих переменных), в то время как в рассматриваемой нами термодинамике гамильтониан отсутствует вовсе.

3.5. Функция Планка. Чтобы изучать фазовые переходы, следует, как и в случае термодинамических потенциалов «энергетического» типа, перейти к интенсивным переменным, т. е. к псевдокоординатам. В силу однородности лагранжева многообразия это можно сделать, только если зафиксировать какую-либо экстенсивную переменную. Как и ранее, будем считать фиксированным число $N$ частиц в системе и перейдем от координат $(U, V)$ к координатам $\beta, \beta p$.

Как и всегда при переходе к сопряженным координатам на лагранжевом многообразии, нужно совершить над действием преобразование Лежандра, т. е. в данном случае перейти к функции

$$
\Phi=S-\beta U-\beta p V
$$

на нашем лагранжевом многообразии и выразить ее через координаты $\beta$ и $\beta p$. По общим формулам дифференциал этой функции равен

$$
d \Phi=-U d \beta-V d(\beta p) .
$$

Удобно совершить замену псевдокоординат $(\beta, \beta p) \mapsto(\beta, p)$ (при замене псевдокоординат, сопровождаемой ассоциированной заменой псевдоимпульсов, действие не меняется). Тогда выражение для $d \Phi$ приобретает вид

$$
d \Phi=-H d \beta-(\beta V) d p,
$$

т. е. при таких координатах сопряженными псевдоимпульсами служат энтальпия $H$ и «объем $\beta V$ на градус температуры». Функция $\Phi$ хорошо известна в термодинамике; это так называемая функиия Планка, которая выражается через термодинамический потенциал Гиббса формулой

$$
\Phi=-G / T=-\beta G .
$$

3.6. Фазовый переход первого рода. Точки фазового перехода первого рода в системе определяются условием равенства значений функции Планка на 
двух ветвях лагранжева многообразия над данной точкой $(\beta, p)$ (что́ выражается «правилом равенства площадей»). В этих точках при изменении параметров $(\beta, p)$ происходит «перескок» системы на ветвь с бо́льшим значением $\Phi$, что и интерпретируется как фазовый переход первого рода.

В силу формулы (40) совпадение значений функции Планка на ветвях лагранжева многообразия над данной точкой $(\beta, p)$ эквивалентно совпадению значений в тех же точках термодинамического потенциала Гиббса. Иными словами, как и следовало ожидать, предсказываемая указанным правилом точка фазового перехода не зависит от конкретного выбора представления системы в интенсивных координатах, а максимизация функции Планка эквивалентна минимизации потенциала Гиббса.

ЗАмЕЧАНИЕ 16. Заметим, что в силу формулы (39) максимизация функции Планка (т. е. минимизация потенциала Гиббса) эквивалентна условной максимизации энтропии при фиксированных $U$ и $V$ ( $\beta$ и $\beta p$ играют роль множителей Лагранжа). Таким образом, имеет место двойственность

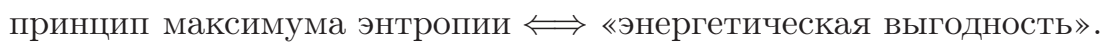

\section{§4. Применение туннельного оператора к термодинамике}

4.1. Кинетические коэффициенты и мера. Оказывается, на лагранжевом многообразии $\Lambda$, определяющем возможные состояния термодинамической системы, имеется (определенная с точностью до постоянного множителя) естественная мера $\mu$. Эта мера может быть определена следующим образом через так называемые кинетические коэффициенты. Предположим, что при заданном $\alpha_{0} \in \Lambda$ наша система находится в термодинамическом равновесии с окружающей средой и затем мы вывели систему из равновесия, слегка сдвинувшись из точки $\alpha_{0}: \alpha=\alpha_{0}+x$, где $x$ - малое изменение параметров (т. е. в локальных координатах на $\Lambda$ компоненты $x_{1}, \ldots, x_{n}$ вектора $x$ малы). Тогда, как известно, система будет стремиться вернуться в равновесное состояние (т. е. состояние с $x=0$, в котором совокупная энтропия $\mathfrak{S}(x)$ системы и окружающей среды максимальна) в соответствии с уравнениями

$$
\dot{x}_{j}=\sum_{j=1}^{n} \gamma_{j k} \frac{\partial \mathfrak{S}}{\partial x_{k}},
$$

где $\gamma_{j k}$ - кинетические коэффициенть [1], [9]. Известно, что матрица $\left\{\gamma_{j k}\right\}$ симметрична (это соотношения Онзагера) и положительно определена. Уравнения (41) можно трактовать как нахождение точки максимума $x=0$ энтропии $\mathfrak{S}(x)$ методом наискорейшего подъема.

Выясним, как изменяются коэффициенты $\left\{\gamma_{j k}\right\}$ при замене координат $y=$ $y(x)$. В новых координатах

$$
\dot{y}_{j}=\sum_{j=1}^{n} \widetilde{\gamma}_{j k} \frac{\partial \mathfrak{S}}{\partial y_{k}}, \quad \widetilde{\gamma}=\frac{\partial y}{\partial x} \gamma\left(\frac{\partial y}{\partial x}\right)^{\prime}
$$

где штрих означает транспонирование. Таким образом, $\left\langle\gamma^{-1} d x, d x\right\rangle=\left\langle\widetilde{\gamma}^{-1} d y, d y\right\rangle$, т. е. матрица $\gamma^{-1}$ задает корректно определенную метрику на лагранжевом многообразии $\Lambda$ в точке $\alpha_{0}$. 
Через $\mu$ обозначим соответствующий этой метрике риманов элемент объема на $\Lambda$. Эту меру и будем использовать в конструкции туннельного канонического оператора на $\Lambda$.

ЗАмЕчАНИЕ 17. При изменении единиц измерения времени все кинетические коэффициенты умножатся на одну и ту же константу, а значит, на константу умножится и мера. Однако для наших целей это несущественно, поскольку нас интересует лишь статистический потенциал - логарифм выражения, задаваемого каноническим оператором, а при умножении меры на константу он изменится на аддитивную константу, которая в определении потенциала несущественна.

Возможность практического определения кинетических коэффициентов связана с тем, что они имеют физический смысл макроскопически наблюдаемых кинетических величин, таких, как теплопроводность, коэффициент диффузии, электропроводность, коэффициент химического сродства, коэффициент термодиффузии, коэффициент Дюфура и др.

4.2. О построении лагранжева многообразия для смесей веществ. Предположим, что мы имеем дело со смесью $k$ газов, возможные термодинамические состояния каждого из которых по отдельности описываются некоторым лагранжевым многообразием $L_{j}$ в фазовом пространстве $\Phi_{j}=\mathbb{R}^{2 n_{j}}$. Как описать термодинамические состояния смеси? Для этого рассмотрим лагранжево многообразие

$$
\Lambda=L_{1} \times \cdots \times L_{k} \subset M=\Phi_{1} \times \cdots \times \Phi_{k},
$$

представляющее собой прямое произведение лагранжевых многообразий, отвечающих индивидуальным газам, и редуцируем его следующим образом.

Предположим, что среди интенсивных переменных имеются химические потенциалы, на которые химические реакции налагают соотношения вида [11]

$$
\sum_{j=1}^{k} \nu_{i j} \boldsymbol{q}_{j}=0, \quad i=1, \ldots, l,
$$

где $\nu_{i j}$ - некоторые константы. Наличие таких соотношений позволяет понизить размерность рассматриваемого лагранжева многообразия $\Lambda$ в общем положении на $l$ единиц. В общем виде соответствующая конструкция выглядит так: задано подмногообразие $M_{0} \subset M$ конфигурационного пространства, выделенное связями типа (44). Рассмотрим пересечение многообразия $\Lambda$ с $\pi^{-1}\left(M_{0}\right)$, где $\pi: T^{*} M \rightarrow M-$ естественная проекция. Предположим, что это гладкое многообразие (это заведомо так для неособой карты на $\Lambda$ ). Вложение $M_{0} \subset M$ порождает (с помощью отображения обратного образа для дифференциальных форм) естественную проекцию

$$
\Pi: \pi^{-1}\left(M_{0}\right) \longrightarrow T^{*} M_{0},
$$

и если $\Pi\left(\Lambda \cap \pi^{-1}\left(M_{0}\right)\right)$ - гладкое многообразие, то оно будет лагранжевым многообразием размерности $n-l$.

В координатах в нашем примере это выглядит следующим образом. Сделаем линейную замену интенсивных переменных таким образом, чтобы соотношения (44) приобрели вид

$$
\boldsymbol{q}_{i}=0, \quad i=1, \ldots, l .
$$


Разобьем координаты $\boldsymbol{q}$ на две группы: $\boldsymbol{q}=\left(\boldsymbol{q}^{\prime}, \boldsymbol{q}^{\prime \prime}\right)$, где $\boldsymbol{q}^{\prime}=\left(\boldsymbol{q}_{1}, \ldots, \boldsymbol{q}_{l}\right)$. В неособой карте лагранжево многообразие задается формулами

$$
\boldsymbol{p}^{\prime}=\frac{\partial S}{\partial \boldsymbol{q}^{\prime}}\left(\boldsymbol{q}^{\prime}, \boldsymbol{q}^{\prime \prime}\right), \quad p^{\prime \prime}=\frac{\partial S}{\partial \boldsymbol{q}^{\prime \prime}}\left(\boldsymbol{q}^{\prime}, \boldsymbol{q}^{\prime \prime}\right) .
$$

Тогда «приведенное» лагранжево многообразие $\Pi\left(\Lambda \cap \pi^{-1}\left(M_{0}\right)\right)$ с учетом связей (45) задается формулами

$$
\begin{gathered}
\boldsymbol{p}^{\prime \prime}=\frac{\partial S}{\partial \boldsymbol{q}^{\prime \prime}}\left(0, \boldsymbol{q}^{\prime \prime}\right) . \\
\text { ЛитеРАТУРА }
\end{gathered}
$$

[1] Л. Д. Ландау, Е. М. Лифшиц, Статистическая физика, ГИТТЛ, М.-Л., 1951.

[2] Е. М. Фейнман, А. Хибс, Квантовая механика и интегралы по траекториям, Мир, М., 1968.

[3] В. П. Маслов, Теория возмущений и асимптотические методь, Изд-во МГУ, М., 1965.

[4] В. П. Маслов, Асимптотические методы и теория возмущений, Наука, М., 1988.

[5] В. И. Арнольд, Математические методь классической механики, Наука, М., 1989.

[6] В. П. Маслов, “Аналитическое продолжение асимптотических формул и аксиоматика термодинамики и квазитермодинамики", Функи. анализ и его прил., 28:4 (1994), 28-41.

[7] В. П. Маслов, "Геометрическое квантование термодинамики, фазовые переходы и асимптотика в критических точках", Матем. заметки, 56:3 (1994), 155-156.

[8] Р. Фейнман, Статистическая механика, Мир, М., 1975.

[9] S. R. de Groot, Thermodynamics of Irreversible Processes, North-Holland, Amsterdam, 1952.

[10] R. Kubo, Thermodynamics, North-Holland, Amsterdam, 1968.

[11] A. Münster, Chemische Thermodynamik, Akademie-Verlag, Berlin, 1969.

Институт проблем механики РАН

Московский государственный университет

e-mail: v.p.maslov@mail.ru

Институт проблем механики РАН

e-mail: nazaikinskii@yandex.ru
Поступило в редакцию 28 марта 2006 г. 